\title{
ARTICLE \\ Mitochondrial DNA in the tumour microenvironment activates neutrophils and is associated with worse outcomes in patients with advanced epithelial ovarian cancer
}

Kelly L. Singel ${ }^{1}$, Kassondra S. Grzankowski ${ }^{2,3}$, A. N. M. Nazmul H. Khan ${ }^{4}$, Melissa J. Grimm ${ }^{4}$, Anthony C. D’Auria ${ }^{4}$, Kayla Morrell ${ }^{5}$, Kevin H. Eng ${ }^{5}$, Bonnie Hylander ${ }^{1}$, Paul C. Mayor ${ }^{2}$, Tiffany R. Emmons ${ }^{1}$, Nikolett Lénárt ${ }^{6}$, Rebeka Fekete ${ }^{6}$, Zsuzsanna Környei ${ }^{6}$, Uma Muthukrishnan ${ }^{7}$, Jonathan D. Gilthorpe ${ }^{7}$, Constantin F. Urban ${ }^{8}$, Kiyoshi Itagaki ${ }^{9}$, Carl J. Hauser ${ }^{9}$, Cynthia Leifer $^{10}$, Kirsten B. Moysich ${ }^{11}$, Kunle Odunsi ${ }^{1,2,12}$, Ádám Dénes ${ }^{6}$ and Brahm H. Segal ${ }^{1,4,13}$

BACKGROUND: Advanced cancer causes necrosis and releases damage-associated molecular patterns (DAMPs). Mitochondrial DAMPs activate neutrophils, including generation of neutrophil extracellular traps (NETs), which are injurious, thrombogenic, and implicated in metastasis. We hypothesised that extracellular mitochondrial DNA (mtDNA) in ascites from patients with epithelial ovarian cancer (EOC) would correlate with worse outcomes.

METHODS: Banked ascites supernatants from patients with newly diagnosed advanced EOC were analysed for mtDNA, neutrophil elastase, and activation of healthy donor neutrophils and platelets. TCGA was mined for expression of SELP and ELANE.

RESULTS: The highest quartile of ascites mtDNA correlated with reduced progression-free survival (PFS) and a higher likelihood of disease progression within 12-months following primary surgery $(n=68$, log-rank, $p=0.0178)$. NETs were detected in resected tumours. Ascites supernatants chemoattracted neutrophils, induced NETs, and activated platelets. Ascites exposure rendered neutrophils suppressive, based on abrogation of ex vivo stimulated T cell proliferation. Increased SELP mRNA expression correlated with worse overall survival ( $n=302$, Cox model, $p=0.02$ ).

CONCLUSION: In this single-centre retrospective analysis, ascites mtDNA correlated with worse PFS in advanced EOC. Mitochondrial and other DAMPs in ascites may activate neutrophil and platelet responses that facilitate metastasis and obstruct anti-tumour immunity. These pathways are potential prognostic markers and therapeutic targets.

British Journal of Cancer (2019) 120:207-217; https://doi.org/10.1038/s41416-018-0339-8

\section{BACKGROUND}

Epithelial ovarian cancer (EOC) is the leading cause of death from gynaecological malignancies and the second most common gynaecological cancer in the United States. In an analysis of 1,895 patients with stage III EOC treated with primary surgery and standard chemotherapy, the median progression-free survival (PFS) was 17 months and the median overall survival (OS) was 45 months. ${ }^{1}$ Prognostic factors for advanced EOC include age, performance status, residual tumour volume, tumour histology, and serum CA-125 levels. ${ }^{1,2}$ The immune responses in the tumour microenvironment can also influence clinical outcome and are potential targets for therapeutic modulation. Tumour-infiltrating $\mathrm{T}$ cell accumulation and high $\mathrm{CD}^{+}$to regulatory $\mathrm{T}$ cell ratio predicted better outcomes, while increased regulatory $\mathrm{T}$ cell accumulation predicted worse outcomes in patients with advanced EOC. ${ }^{3-5}$ The tumour microenvironment of EOC is inflammatory as well as immunosuppressive, and characterised by the accumulation of ascites, and mature and immature myeloid cells, cytokines, and chemokines. ${ }^{6-11}$ Cui et al. ${ }^{12}$ reported that myeloid cell accumulation in EOC triggered acquisition of stem cell-like features in cancer cells, increased metastatic potential, and was also associated with worse outcomes. Although clinical variables such as tumour stage and histology cannot be modified, the immune responses shaping the tumour microenvironment are potential novel prognostic biomarkers and therapeutic targets.

Neutrophils and platelets become activated as emergency responders to infection and injury, and co-migrate to sites of injury. ${ }^{13}$ While initial responses are critical for defence against infection, control of bleeding and promotion of wound repair, these responses can accelerate tumour progression when active in

\footnotetext{
${ }^{1}$ Department of Immunology, Roswell Park Comprehensive Cancer Center, Buffalo, NY, USA; ${ }^{2}$ Department of Surgery, Division of Gynecologic Oncology, Roswell Park Comprehensive Cancer Center, Buffalo, NY, USA; ${ }^{3}$ Arizona Center for Cancer Care, Phoenix, AZ, USA; ${ }^{4}$ Department of Medicine, Roswell Park Comprehensive Cancer Center, Buffalo, NY, USA; ${ }^{5}$ Department of Biostatistics and Bioinformatics, Roswell Park Comprehensive Cancer Center, Buffalo, NY, USA; ${ }^{6}$ Momentum Laboratory of Neuroimmunology, Institute of Experimental Medicine, Hungarian Academy of Sciences, Budapest, Hungary; ${ }^{7}$ Department of Pharmacology and Clinical Neuroscience, Umeå University, Umeå, Sweden; ${ }^{8}$ Department of Clinical Microbiology, Umeå University, Umeå, Sweden; ${ }^{9}$ Department of Surgery, Beth Israel Deaconess Medical Center, Harvard Medical School, Boston, MA, USA; ${ }^{10}$ Department of Microbiology and Immunology, Cornell University College of Veterinary Medicine, Ithaca, NY, USA; ${ }^{11}$ Department of Cancer Prevention and Control,

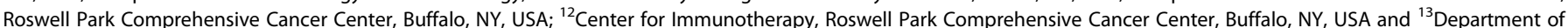
Medicine, Jacobs School of Medicine and Biomedical Sciences, University at Buffalo, Buffalo, NY, USA Correspondence: Brahm H. Segal (brahm.segal@roswellpark.org)
}

Received: 24 July 2018 Revised: 9 October 2018 Accepted: 25 October 2018

Published online: 6 December 2018 
the tumour microenvironment. Labelle et al. $^{14}$ showed that platelet-derived chemokines CXCL5/7 guide granulocytes to circulating tumour cells. This platelet-granulocyte interaction with tumour cells promotes tumour seeding and metastasis. In patients with EOC, elevated pre-treatment circulating neutrophil counts ${ }^{15}$ and high neutrophil-to-lymphocyte ratio ${ }^{16}$ correlated with poor outcomes. Paraneoplastic thrombocytosis predicted worse outcomes in patients with a number of solid tumours, including newly diagnosed advanced EOC. ${ }^{17,18}$ In addition, platelet infiltration into tumours after anti-angiogenic therapy withdrawal led to enhanced tumour rebound. ${ }^{19}$

Mitochondrial DAMPs (mtDAMPs), which include mitochondrial DNA (mtDNA) and formylated peptides, are released following traumatic injury and activate neutrophils through ligation of tolllike receptor 9 (TLR9) and formylated peptide receptor. We previously observed that mtDAMPs stimulated the generation of neutrophil extracellular traps (NETs). ${ }^{20}$ NETosis is a distinct mode of neutrophil death characterised by the breakdown of membranes and extracellular release of chromatin and granular constituents. ${ }^{21}$ NETosis results in the release of products that can augment antimicrobial host defence but also cause tissue injury. ${ }^{22}$ NETs have been shown to promote thrombosis, likely through the release of extracellular chromatin ${ }^{23-25}$ and tissue factor, $^{26}$ while P-selectin also promotes NETosis. ${ }^{27}$ In addition, NETosis is implicated in both cancer-associated thrombosis ${ }^{24}$ and acceleration of metastasis. ${ }^{28,29}$ Taken together, different experimental models point to injury and release of DAMPs in the tumour microenvironment stimulating neutrophilic and prothrombogenic responses that can enhance tumour progression. We hypothesised that mtDNA in the tumour microenvironment activates neutrophil and platelet wound repair responses and correlates with worse outcomes in patients with newly diagnosed advanced EOC.

Previously, we reported that the combination of high tumour necrosis factor-alpha and IL-6 in the ascites was associated with reduced PFS in patients with newly diagnosed advanced EOC. ${ }^{30}$ This signature suggested an interaction between proinflammatory pathways driving tumour progression and resistance to chemotherapy in advanced EOC. In addition, we observed that granulocytes isolated from the ascites suppressed stimulated $T$ cell proliferation ex vivo. ${ }^{31}$ Here we investigated mtDNA in the ascites as a potential driver for proinflammatory pathways and reduced survival. Together, our results suggest that products of injury (e.g., mtDNA), and the subsequent neutrophil and platelet responses, are potential prognostic biomarkers and novel therapeutic targets in patients with advanced EOC.

\section{METHODS}

Patients and Specimens

This study was approved by the Institutional Review Board (IRB) of Roswell Park Comprehensive Cancer Center (Roswell Park), Buffalo, NY, and was in compliance with federal and state requirements. All participants gave informed consent to use samples for research. Ascites was collected and processed from patients with newly diagnosed advanced EOC, as previously described. ${ }^{30}$ The medical records of these patients were retrospectively reviewed for PFS and OS calculated from time of diagnosis. Recurrence was defined by objective criteria based on CT scan imaging. PFS was the interval between diagnosis to disease progression, recurrence, or death. OS was the interval from diagnosis to date of death or censored at date of last follow-up. Banked ascites supernatants from patients with cirrhosis and without cancer were provided by Dr. Thomas Russo (Jacobs School of Medicine and Biomedical Sciences, University at Buffalo).
Mice

Mice with a targeted disruption of the $\mathrm{p} 47^{\text {phox }}$ gene $\left(\mathrm{p} 47^{\text {phox-/-}}\right.$ ) have a defective phagocyte NADPH oxidase (NOX2), rendering phagocytes incapable of generating measurable superoxide. p47 ${ }^{\text {phox-1- }}$ mice were backcrossed 14 generations in the C57BL/ $6 \mathrm{Ncr}$ background. A p47 $7^{\text {phox-1-}}$ mouse breeding colony is established at Roswell Park. C57BL/6Ncr mice were purchased

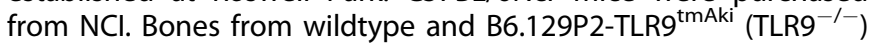
mice (backcrossed 11 generations in the C57BL/6 background) were obtained from Dr. Cynthia Leifer (Cornell University College of Veterinary Medicine, Ithaca, NY). Female mice (age 6-8 weeks) were used in all experiments. Animals were bred and maintained under specific pathogen-free conditions at the animal care facility at Roswell Park and used in compliance with all relevant laws and institutional guidelines under a protocol approved by the Institutional Animal Care and Use Committee.

Purification of murine bone marrow neutrophils

Bone marrow-purified neutrophils (BM-PMNs) were isolated from 10-week-old female C57BL/6Ncr wildtype (WT) and p47 $7^{\text {hox-1- }}$ mice. Briefly, long bones were harvested from each mouse and flushed with DPBS using a $26 \mathrm{G}$ needle. Single-cell suspensions were generated and centrifuged on Percoll (\#P1644, MilliporeSigma, St. Louis, MO, USA) density gradients to isolate BM-PMNs. The purity of BM-PMNs was $>90 \%$ based on cytology.

Activation of murine bone marrow-derived dendritic cells Bone marrow was harvested from the long bones of WT and $\mathrm{TLR9}^{-1-}$ mice as described. To generate bone-marrow-derived dendritic cells (BMDCs), single-cell suspensions were generated and cultured in RPMI 1640 complete media containing murine GM-CSF $(20 \mathrm{ng} / \mathrm{ml})$ for 6-7 days. Media were refreshed every 2 days. For in vitro activation, BMDCs were treated with mtDNA $(25 \mu \mathrm{g} / \mathrm{ml})$ or $\mathrm{CpG}$ sequences $(2 \mu \mathrm{M})$ for $24 \mathrm{~h}$ in media without GMCSF. Polymixin B $(10 \mu \mathrm{g} / \mathrm{ml})$ was added with $\mathrm{mtDNA}$ as a specificity control. Flow cytometry analysis was conducted on a BD LSR II FACScan (Becton, Dickinson and Company, Franklin Lakes, NJ, USA). Forward scatter versus side scatter gating was set to include all non-aggregated cells from at least 20,000 events collected per sample.

\section{Isolation of donor neutrophils}

Peripheral blood was collected from healthy donors after receiving written informed consent under an IRB-approved protocol. Neutrophils were isolated from peripheral blood by 2-step density gradient separation. First, blood was centrifuged on Histopaque (\#1119, MilliporeSigma) to separate the granulocyte-rich fraction. Then neutrophils were isolated from the granulocyte-rich fraction by a second centrifugation on Percoll density gradients. The purity of neutrophils was $>90 \%$ based on cytology and $\mathrm{CD} 33^{+} \mathrm{CD} 15^{+}$ expression.

\section{Extraction of mitochondrial DAMPs, mitochondrial DNA, and} genomic DNA

De-identified remnant benign margins of resected liver from patients undergoing surgery for hepatic tumours were used as an abundant source of genomic DNA (gDNA) and mtDNA under an IRB-approved protocol. Mitochondrial DAMPs were isolated as previously described. ${ }^{32}$ Briefly, $200 \mathrm{mg}$ of liver was sonicated on ice at $100 \%$ amplitude (30-sec each time with 30 -s intervals, 10 times). The disrupted mitochondrial suspensions were centrifuged at $12,000 \mathrm{~g}$ for $10 \mathrm{~min}$ and at $10,000 \mathrm{~g}$ for $30 \mathrm{~min}$. Supernatants were used in experiments as mtDAMPs; the yield of mtDAMPs is similar to that recovered in vivo in experimental 5\% liver injury models. mtDNA was extracted using the mtDNA Extractor CT kit (\#291-55301, Wako Chemicals, Richmond, VA, USA) following the manufacturer's protocol. gDNA was extracted using QIAmp DNA 
Mini Kit following the manufacturer's protocol (\#51304, Qiagen, Germantown, MD, USA).

Quantification of mitochondrial DNA and genomic DNA Total DNA was extracted from ascites supernatants using the DNA purification kit (\#51106, Qiagen) following the manufacturer's protocol. Purified mtDNA from human liver, SYBR Green ER SuperMix kit (\#11760, Thermo Fisher Scientific, Waltham, MA, USA), and mitochondrial-specific human cytochrome $B$ primers (forward 5'-ATGACCCCAATACGCAAAAT-3' and reverse 5'CGAAGTTTCATCATGCGGAG-3) for real-time quantitative PCR (qPCR) were used to evaluate mtDNA in total DNA isolated from ascites. ${ }^{32}$ A similar method was applied to evaluate gDNA in ascites. qPCR analysis was conducted on the ABI Fast Real-Time instrument (Thermo Fisher Scientific).

Detection of neutrophil extracellular traps ex vivo BM-PMNs purified from WT and $\mathrm{p} 47^{\text {phox-I- }}$ mice and circulating neutrophils from healthy donors were evaluated for NETosis. NETs were detected by immunofluorescent confocal microscopy using previously published methods. ${ }^{33}$ Neutrophils were exposed to phorbol 12-myristate 13-acetate (PMA, $100 \mathrm{nM}$ ) as a positive control, mtDAMPs, or ascites supernatants for $1 \mathrm{~h}$. NETs were identified by immunofluorescent confocal microscopy based on extracellular stretches of DNA, as previously described. ${ }^{33}$ Immunostaining and confocal microscopy were performed as described in supplementary information. The major endpoints evaluated were the presence of NETs (yes/no) or NE levels in supernatants by ELISA.

Immunostaining of resected EOC from patients

NETs were assessed in resected tumour from patients who underwent primary surgery for advanced EOC at Roswell Park. 5 $\mu \mathrm{m}$ FFPE sections were deparaffinised, rehydrated in decreasing concentrations of ethanol, and subjected to antigen retrieval by heating in $10 \mathrm{mM}$ citrate buffer $(\mathrm{pH} \mathrm{6.0)}$ for $10 \mathrm{~min}$. Specimens were blocked with a solution of $2 \%$ bovine serum albumin and $5 \%$ donkey serum in PBS $+0.1 \%$ Triton $\mathrm{X}-100$ for $1 \mathrm{~h}$ at room temperature. Immunostaining and confocal microscopy were performed as described in supplementary information.

Evaluation of neutrophil chemotaxis in response to Ascites in vitro A 96-well plate system with top and bottom chambers separated by a transmembrane with $3 \mu \mathrm{m}$ pores was used. Neutrophils from healthy donors $\left(10^{5}\right.$ cells/well) were plated in the top chamber. To attract the neutrophils, 5\% mtDAMPs prepared from human liver or $10 \%$ ascites supernatants were added to the bottom chamber and incubated for $1 \mathrm{~h}$ at $37^{\circ} \mathrm{C}$ in $5 \% \mathrm{CO}_{2}$. Neutrophils were pretreated with $0.1 \%$ DMSO or $3 \mu \mathrm{M}$ sphingosine kinase inhibitor (SKI). Adherent cells were detached from the transmembrane with lysis buffer and combined with the neutrophils that had migrated into the bottom chamber. Neutrophils were labelled with CyQuant GR dye (\#C7026, Thermo Fisher Scientific) and evaluated against a standard curve to determine number of migrated cells.

Identification of platelet microparticles by flow cytometry To isolate and prepare platelet microparticles (PMPs), ascites supernatants were sequentially centrifuged at $500 \times g$ for 10 minutes, $2000 \mathrm{~g}$ for 10 minutes, and $25,000 \times g$ for 20 minutes. PMPs ranging from 500 to $1000 \mathrm{~nm}$ were observed in the $25,000 \times g$ pellet. Samples were stained with anti-CD41a (\#12-0419, Thermo Fisher Scientific) and visualised on a BD Fortessa flow cytometer (Becton, Dickinson and Company).

Isolation of platelets from donor and murine peripheral blood Platelets were isolated from healthy donor peripheral blood or blood from the right cardiac ventricle of anesthetised male C57BL/ 6 mice (breeding at the Institute of Experimental Medicine,
Budapest, Hungary). Blood was anticoagulated with $10 \%$ volume of acid citrate dextrose (ACD). Methods are described in more detail in supplementary information.

Statistical analysis

All statistical analyses were performed using the R 3.4.0 statistical computing language. A nominal significance threshold of 0.05 was used unless otherwise specified. Statistical testing included Student's $t$ test, $x^{2}$ and Fisher's exact tests, and Kaplan-Meier survival analysis with log-rank testing. The multivariate analysis comprised FIGO stage, categorised as early (I, II, or IIIA/B) or late (IIIC or IV), histological grade, debulking status (optimal defined by $1 \mathrm{~cm}$ margin and R0 defined as no macroscopic residual disease), and platinum-sensitive versus refractory disease. Patients were censored from the multivariate analysis if they were reported as alive with no evidence of disease. Restricted mean survival (RMS) curves described by Eng et al. ${ }^{34,35}$ were computed using ascites NE levels as a continuous variable.

TCGA provisional RNA Seq V2 RSEM data were downloaded from cBioportal for ovarian serous cystadenocarcinoma and mined for ELANE, SELP, and ITGB3 expression. OS $(n=302)$ data was analysed for each primary tumour sample from cBioportal. Using Cox-Proportional Hazards Regression model, RMS curves were created using quantiles of the genes.

\section{RESULTS}

High ascites mtDNA and neutrophil elastase is associated with reduced progression-free survival following primary surgery Banked ascites supernatants $(n=80)$ from patients with newly diagnosed EOC were analysed for mtDNA. Twelve patients were excluded based on early stage (I or II) or non-EOC histology. We observed substantial variability in the mtDNA concentration in ascites supernatants $(n=68$; mean $298 \mathrm{ng} / \mu \mathrm{L}$, range $<1$ to 3139 $\mathrm{ng} / \mu \mathrm{L}$ ) (Fig. 1a). We found that levels of mtDNA (log-rank, $p=$ 0.0178 , Fig. 1b), but not genomic DNA (gDNA, log-rank, $p=0.839$, Fig. 1c), were associated with reduced median PFS. This effect was observed over a range of mtDNA percentiles (top tertile, quartile, and quintile). The $m \mathrm{TDNA}^{\text {high }}$ group was specified as patients with ascites mtDNA >75th quartile, and the mtDNA ${ }^{\text {low }}$ group was specified as patients with ascites mtDNA $<75^{\text {th }}$ quartile (Table 1). The 12-month PFS was the primary clinical endpoint. 23/68 (34\%) patients were in the mtDNA ${ }^{\text {high }}$ group. Twenty-six percent (26\%) of patients in the mtDNA ${ }^{\text {high }}$ and $16 \%$ in the $m t D N A^{\text {low }}$ sub-groups were Stage IV. The mean age at diagnosis was 66.0 years in the mtDNA ${ }^{\text {high }}$ group and 60.4 years in the mtDNA ${ }^{\text {low }}$ group. Histology and grade were not significantly different between the two groups with majority being high-grade (3) serous ovarian cancer (HGSOC; accounts for $70 \%$ of EOC cases). In addition, we censored 7 patients based on their clinical status reported as alive with no evidence of disease, thus $61 / 80$ patients were included in the multivariate analysis. Using the Cox model, the median PFS in the $\mathrm{mtDNA}^{\text {high }}$ and $\mathrm{mtDNA}{ }^{\text {low }}$ groups was $7.6(\mathrm{HR}=1.86,95 \% \mathrm{Cl}: 5.61-$ $15.80)$ vs. $15.2(\mathrm{HR}=0.54,95 \% \mathrm{Cl}: 10.43-19.00)$ months, respectively $(p=0.26)$.

Since mtDAMPs activate neutrophils, including degranulation $^{32}$ and generation of NETs, ${ }^{20}$ we evaluated two products of neutrophil degranulation, neutrophil elastase (NE) and myeloperoxidase, in banked ascites supernatants. Patients with the highest levels of ascites NE had a significantly lower likelihood of achieving PFS at 12 months ( $n=67$, log-rank, $p<0.001$ ) (Table 2$)$, while myeloperoxidase levels did not correlate with outcome. Considering the ascites NE levels as a continuous variable, we evaluated the restricted mean survival (RMS) curve (Supplemental Fig. 1). The RMS curve shows that with higher levels of ascites NE (e.g., $1200 \mathrm{ng} / \mathrm{mL}$ ), patients had a survival of approximately 8.5 months, whereas with lower levels of ascites $\mathrm{NE}$ (e.g., $200 \mathrm{ng} / \mathrm{mL}$ ), patients survived approximately 

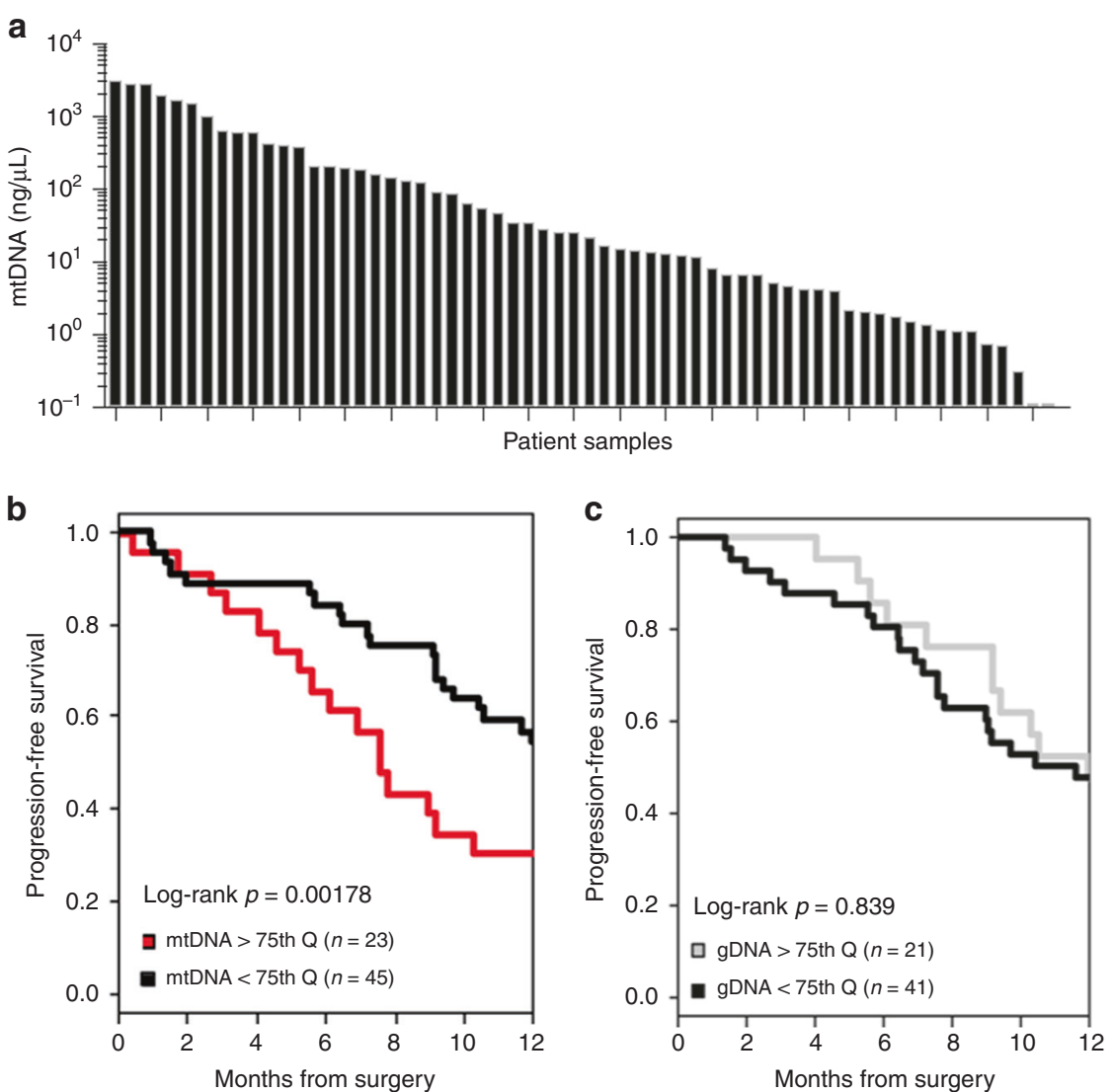

Fig. 1 High ascites mtDNA levels are associated with reduced progression-free survival following primary surgery. a-c High ascites levels of mtDNA, but not gDNA, are associated with reduced median PFS. Banked ascites supernatants from patients with newly diagnosed advanced EOC were analysed for mtDNA and gDNA. a Variability exists between ascites mtDNA ( $\mathrm{ng} / \mu \mathrm{L})$ levels as measured by qPCR $(n=68)$. b High mtDNA levels as defined by the upper $75^{\text {th }}$ quartile (red line) are associated with reduced PFS as compared to the lowest $75^{\text {th }}$ quartile (black line) of mtDNA ( $n=68$, log-rank, $p=0.0178)$. c Ascites gDNA levels are not associated with PFS $(n=62, \log$-rank, $p=0.839)$

11.5 months. However, these are the extremes of ascites NE levels, and the relationship was not linear. The RMS curve highlights the complexity of the relationship between ascites NE levels and survival. Together, these results point to ascites mtDNA and NE levels at diagnosis of advanced EOC as potential prognostic biomarkers.

Ovarian cancer ascites is chemoattractive to neutrophils and induces neutrophil extracellular traps and suppressive neutrophils Since NETs have been visualised in solid tumours, ${ }^{29,36,37}$ we determined whether NETosis occurs in tumour-infiltrating neutrophils at primary surgery for EOC. Tumour-infiltrating neutrophils were uncommonly observed, but both intact neutrophils (Fig. 2a-d) and NETs (Fig. 2e-h) were observed in tumours from $4 / 5$ patients.

In ascites from patients with newly diagnosed advanced EOC, the predominant cellular population was leukocytes $\left(C D 45^{+}\right)$ (mean 92.9\%, $n=9 \mathrm{HGSOC}$ ) (Fig. 2i). As we previously reported, $\mathrm{CD}^{+} 5^{+}$neutrophils were $\sim 15-20 \%$ of ascites cells. ${ }^{31}$ Since neutrophils are short-lived, ongoing neutrophil recruitment is required to maintain neutrophilic inflammation. Therefore, we evaluated whether ascites supernatants act as a chemoattractant for neutrophils. Using mtDAMPs as a positive control, we found that ascites supernatants stimulated neutrophil chemotaxis (Fig. 2j), and that sphingosine kinase inhibitor (SKI), which inhibits calcium influx, reduced chemotaxis elicited by both mtDAMPs and ascites supernatants.

Next, we evaluated whether ascites supernatants stimulated NETosis. NETosis can occur through NADPH oxidase
(NOX2)-dependent ${ }^{33}$ and -independent pathways. ${ }^{38}$ First, we asked whether mtDAMPs stimulated NOX2 and if NETosis was NOX2-dependent. Using purified BM-PMNs from WT and NOX2deficient $\mathrm{p} 47^{\text {phox } / \text { - }}$ mice, we found that mtDAMPs activated NOX2 as measured by DHR123 fluorescence (Supplemental Fig. 2A). PMA was used as a positive control for NOX2dependent NETosis, ${ }^{39}$ and, as expected, PMA did not induce NETs in p47 $7^{\text {phox-1- }}$ BM-PMNs (Supplemental Fig. 2B, left upper panel). In contrast, mtDAMPs stimulated NETosis in $\mathrm{p} 47^{\text {phox-/- }}$ BM-PMNs, demonstrating NOX2-independent NETosis (Supplemental Fig. 2B, right upper panel). Consistent with mouse BMPMNs and our prior study, ${ }^{20}$ PMA and mtDAMPs induced NETs in neutrophils from healthy donors (Supplemental Fig. 2B, lower panels). Exposure of neutrophils to 3 of 4 ascites supernatants resulted in NETs as visualised by immunofluorescent confocal microscopy (Fig. 2k). Consistent with induction of NETosis, exposure of neutrophils to ascites supernatants led to NE release (Fig. 2l). Heat-inactivating ascites supernatants before neutrophil exposure reduced the amount of $\mathrm{NE}$ released into supernatants (Fig. 2l). Pre-treating ascites supernatants with DNase I to deplete both gDNA and mtDNA also reduced NE release, but to a lesser extent than heat inactivation (Fig. 2l). Thus, mtDAMPs and ascites supernatants induce NETosis through NOX2-independent pathways, and release of NE is abrogated by prior treatment of ascites supernatants with heat and DNase I.

Finally, since we previously observed that purified ascites granulocytes suppressed stimulated T-cell proliferation, ${ }^{31}$ we asked whether ascites supernatants would induce a similar 
Table 1. Ascites mtDNA levels stratified into lowest and highest $75^{\text {th }}$ quartiles are associated with reduced 12-month survival in patients with newly diagnosed advanced EOC

\begin{tabular}{|c|c|c|c|}
\hline & \multicolumn{3}{|l|}{ mtDNA level } \\
\hline & $<75$ th Q & $>75$ th Q & $p$ value \\
\hline$N$ & 45 & 23 & \\
\hline Age, Mean & 60.4 & 66.0 & 0.06 \\
\hline Primary Site & & & 0.26 \\
\hline Ovary & $80 \%$ & $70 \%$ & \\
\hline Fallopian Tube & $0 \%$ & $4 \%$ & \\
\hline Primary Peritoneal & $20 \%$ & $26 \%$ & \\
\hline Stage & & & 0.19 \\
\hline IIIB & $0 \%$ & $4 \%$ & \\
\hline IIIC & $84 \%$ & $70 \%$ & \\
\hline IV & $16 \%$ & $26 \%$ & \\
\hline Grade & & & 0.76 \\
\hline Well Differentiated & $5 \%$ & $5 \%$ & \\
\hline Moderately Differentiated & $14 \%$ & $23 \%$ & \\
\hline Poorly Differentiated & $81 \%$ & $73 \%$ & \\
\hline Histology & & & 0.30 \\
\hline Serous & $82 \%$ & $70 \%$ & \\
\hline Clear Cell & $4 \%$ & $0 \%$ & \\
\hline Endometrioid & $0 \%$ & $4 \%$ & \\
\hline Mucinous & $2 \%$ & $4 \%$ & \\
\hline Mixed & $11 \%$ & $22 \%$ & \\
\hline Debulking & & & 0.73 \\
\hline Optimal & 71\% & $78 \%$ & \\
\hline Sub-optimal & $29 \%$ & $22 \%$ & \\
\hline \multicolumn{4}{|l|}{ Residual Tumour } \\
\hline $\mathrm{RO}^{\mathrm{a}}$ & $2 \%$ & $9 \%$ & 0.26 \\
\hline Not R0 & $98 \%$ & $91 \%$ & \\
\hline Platinum Status & & & 0.23 \\
\hline Sensitive & $58 \%$ & $39 \%$ & \\
\hline Refractory/Resistant & $42 \%$ & $61 \%$ & \\
\hline \multicolumn{4}{|l|}{ Progression-free survival } \\
\hline Events & 40 & 21 & \\
\hline Median PFS [95\% Cl] & $\begin{array}{l}15.18[10.43- \\
19.00]\end{array}$ & $\begin{array}{l}7.57[5.61- \\
15.80]\end{array}$ & 0.26 \\
\hline $\begin{array}{l}\text { PFS to } 12 \text { months (std. } \\
\text { error) }\end{array}$ & $0.55(0.08)$ & $0.30(0.10)$ & 0.002 \\
\hline $\begin{array}{l}\text { PFS to } 24 \text { months (std. } \\
\text { error) }\end{array}$ & $0.22(0.06)$ & $0.17(0.08)$ & 0.28 \\
\hline HR (PFS) & 0.73 & 1.36 & 0.26 \\
\hline Adj $H^{b}$ & 0.54 & 1.86 & 0.04 \\
\hline
\end{tabular}

suppressor phenotype in circulating neutrophils from healthy donors. In a separate cohort of patients, we found that 17 of 22 HGSOC ascites supernatants induced neutrophils to acquire a suppressor phenotype, abrogating ex vivo anti-CD3/CD28stimulated $\mathrm{T}$ cell proliferation (Fig. $2 \mathrm{~m}$ ). Together these results point to ascites inducing neutrophil recruitment and activation, as well as a suppressor phenotype in the tumour microenvironment.
Mitochondrial DAMPs activate DCs through TLR9-dependent and -independent pathways

Zhang et al. ${ }^{32}$ showed that mtDAMPs activated neutrophils through both TLR9 and formylated peptide receptor. We previously showed that immunisation with an adjuvant containing TLR9 and NOD2 ligands significantly prolonged survival in EOC tumour-bearing mice. ${ }^{31}$ Therefore, we asked whether mtDNA activated DCs and whether activation was TLR9-dependent. WT and TLR9 $^{-1-}$ BMDCs were stimulated with mtDNA and CpG sequences, and surface markers for $D C$ activation were assessed. While DC activation by CpG was TLR9-dependent, mtDNA-induced DC activation was variably TLR9-dependent (Supplemental Fig. 3). The mtDNA-induced upregulation of MHC class I and II and CD80 was TLR9-dependent, while upregulation of CD86 was TLR9independent. Addition of polymyxin B did not alter the expression of costimulatory molecules, arguing against an effect by LPS contamination. These results suggest that mtDAMPs in the tumour microenvironment have broad effects on innate immunity with the potential to suppress or enhance anti-tumour immunity.

Ovarian cancer ascites induces rapid platelet activation and aggregation

In unmanipulated ascites collected at primary surgery for EOC, we observed fibrin deposits with varying proportions of embedded tumour cells and neutrophils (Fig. 3a). The presence of neutrophils is indicative of active inflammation as opposed to clotting that can occur as a processing artifact. Platelet concentration in ascites was very low in relation to circulation, possibly because activated platelets rapidly degranulate. Platelets release microparticles during inflammation, which are internalised by neutrophils and amplify neutrophilic inflammation. ${ }^{40,41}$ We identified CD41a ${ }^{+}$ platelet microparticles (PMPs) in ascites supernatants with substantial variability between samples (Fig. 3b, c). Next, we evaluated mechanisms for ascites-induced platelet activation. Platelets from healthy donors were stimulated with ascites supernatants ex vivo (Supplemental Table 1). Ascites supernatants induced robust platelet activation and aggregation as indicated by an over three-fold increase in P-selectin-positive platelets within 30 minutes after stimulation with $6 / 7$ ascites samples (Fig. 3d). Since healthy donors showed some variation in platelet P-selectin levels, we also tested the ability of ascites supernatants to induce activation of platelets isolated from naïve WT mice. Exposure to ascites supernatants induced a profound increase in P-selectin levels and a reduction in CD42d levels in mouse platelets within 15 minutes, confirming the effect seen with donor platelets (Fig. 3e). In addition, cirrhotic ascites supernatants phenocopied the effect seen with EOC ascites supernatants (Supplemental Fig. 4), suggesting that ascites, regardless of disease origin, activates platelets. These results support the notion that platelets are prone to activation outside of the circulation, particularly in response to an acute injury or inflammatory stimuli, and their responses are not specific to tumour-associated factors.

To investigate potential mechanisms for the observed prothrombogenic activity, EOC ascites supernatants were pre-treated with DNase I and/or protease inhibitors for 30 minutes prior to platelet stimulation. Protease inhibitors and DNase I had modest effects on P-selectin upregulation on donor platelets (Fig. 3f) with substantial inter-donor variability, and CD42d downregulation on murine platelets (Fig. $3 \mathrm{~g}$ ). Using murine platelets, we found that protease inhibitors were modestly more effective at preventing platelet activation as induced by ascites supernatants compared to DNase I. This suggests that there are multiple, most likely redundant, pathways through which ascites can be prothrombogenic and induce platelet activation.

Given the cross signalling between activated neutrophils and platelets, we mined TCGA data for mRNA expression of ELANE, $S E L P$, and ITGB3, which encode NE, P-selectin, and integrin beta-3, 
Table 2. Ascites neutrophil elastase stratified into low, intermediate, and high levels predicts reduced progression-free survival within 12 months following surgery

\begin{tabular}{|c|c|c|c|c|}
\hline & \multicolumn{4}{|c|}{ Neutrophil Elastase Level } \\
\hline$N$ & 11 & 52 & 10 & \\
\hline Age, Mean & 65.3 & 61.4 & 62.7 & ns \\
\hline Stage (\% IV) & $9 \%$ & $23 \%$ & $10 \%$ & ns \\
\hline Grade (\% 3) & $81 \%$ & $76 \%$ & $21 \%$ & ns \\
\hline Events & 9 & 49 & 9 & \\
\hline Median PFS $[95 \% \mathrm{Cl}]$ & $16.26[5.88-\mathrm{NA}]$ & $9.56[6.51-15.0]$ & $5.57[0.29-\mathrm{NA}]$ & 0.0328 \\
\hline PFS to 12 months (std. error) & $0.70(0.144)$ & $0.42(0.069)$ & $0.20(0.127)$ & $<0.001$ \\
\hline PFS to 24 months (std. error) & $0.12(0.109)$ & $0.10(0.044)$ & $0.10(0.095)$ & ns \\
\hline $\mathrm{HR}(\mathrm{PFS})$ & 0.41 & 1 & 2.05 & 0.0367 \\
\hline
\end{tabular}

respectively. NE is expressed by neutrophils, P-selectin can be expressed by both platelets and endothelial cells, and integrin beta-3 is expressed by platelets only. Analysing the SELP RMS curve for $\operatorname{HGSOC}(n=302)$ resulted in a statistically significant reduction in OS (Cox model, $p=0.02, \mathrm{HR}=1.14,95 \% \mathrm{Cl}$ : 1.03-1.28) (Fig. 3h). Neither analyses for ELANE nor ITGB3 varied significantly from a constant function. Together, these results show that EOC ascites is highly thrombogenic, and intratumoural P-selectin expression correlates with worse prognosis.

Extracellular histones are present in ovarian cancer ascites with a predominance of $\mathrm{H} 1$

DNA is packed around core histones (e.g., $\mathrm{H} 2 \mathrm{~A} / \mathrm{B}, \mathrm{H} 3, \mathrm{H} 4$ ) to form the nucleosome, and linker histone $\mathrm{H} 1$ family members bind to nucleosomes to stabilise higher-order chromatin structure. Extracellular histones are released during NETosis and cellular injury and are thrombogenic. We therefore evaluated the histone composition in ascites and its effects on platelet activation. $\mathrm{H} 1$ and $\mathrm{H} 3$ were observed in both untreated ascites supernatants $(500 \mathrm{~g})$ and supernatants from ascites centrifuged at $10,000 \times \mathrm{g}$ (Supplemental Figure 5A-G). $\mathrm{H} 1$ was present in all ascites supernatants tested $(n=9)$ and was not depleted by centrifugation. This observation indicates that $\mathrm{H} 1$ was not associated with membranes and other particles that sediment with centrifugation. Conversely, H3 was only detected in $4 / 9$ of the ascites supernatants and, with the exception of one sample, was not depleted by centrifugation. The concentration of $\mathrm{H} 1$ was 10 -fold higher than the concentration of $\mathrm{H} 3$ in ascites supernatants. The mean nucleosome enrichment factor in ascites supernatants was similar to normal serum, though substantial interpatient variability in ascites supernatants was observed. Four of nine (4/9) ascites supernatants had enriched nucleosomes over normal serum levels (Supplemental Figure $5 \mathrm{H}$ ). There were no significant differences between nucleosome enrichment in EOC patient sera versus normal serum.

Finally, we compared the effects of the ascites supernatants, $10,000 \times g$ supernatants, and $10,000 \times g$ pellets on platelet activation. All treatments significantly upregulated P-selectin on donor platelets (Supplemental Figure 6). In murine platelets, the $10,000 \times g$ supernatants induced upregulation of P-selectin (2/4 ascites), whereas none of the $10,000 \times g$ pellets caused upregulation of P-selectin (Supplemental Figure 7). Since we could not achieve complete depletion of histones from ascites, we cannot make conclusions about whether histones are required for ascitesinduced platelet activation. The ability of soluble and sedimented ascites fractions to activate platelets suggests a number of redundant pro-thrombogenic pathways in ascites.

\section{DISCUSSION}

We previously observed that the volume of ascites at initial diagnosis of EOC correlated with worse PFS and OS, and that intraperitoneal administrations of murine ascites supernatants accelerated EOC progression in vivo. ${ }^{42}$ These results pointed to ascites driving tumour progression. Our results presented here point to mtDNA, and the subsequent neutrophil and platelet responses, as potential prognostic biomarkers and novel therapeutic targets in patients with advanced EOC. In patients with newly diagnosed advanced EOC treated at Roswell Park, the highest quartile of ascites mtDNA was associated with significantly shorter median PFS and a higher likelihood of disease progression within 12-months following primary surgery. Our studies focused on mtDNA and did not address the prognostic value of other DAMPs. Patients with the highest ascites NE levels had significantly shorter median PFS and a higher likelihood of disease progression within 12-months following primary surgery, but the relationship between NE levels and PFS was not linear. We measured NE in banked ascites supernatants by ELISA, which is not a direct measure of NETosis, but a marker of released neutrophil granular products that can occur through NETosis, degranulation, and neutrophil death. Importantly, the mtDNA and NE signatures were significant when restricted to a 12-month window after surgery-an interval that corresponds to clinically defined chemotherapy-refractory disease. This database is from a retrospective analysis from a single center and should therefore be considered exploratory. A larger study of prospectively enrolled patients with newly diagnosed advanced EOC is underway and will test validation of these findings.

TCGA analysis showed that increased mRNA expression of SELP in $\mathrm{HGSOC}$ resected at primary surgery associated with worse OS. Prior studies have shown that ascites is pro-thrombogenic and that thrombin can facilitate tumour invasion through modulation of macrophage function. ${ }^{43,44}$ In addition, Labelle et al. ${ }^{14}$ showed that granulocyte-platelet interactions promoted early metastatic niche that facilitated metastatic seeding and progression. This study focused on the early events promoting metastasis in an 

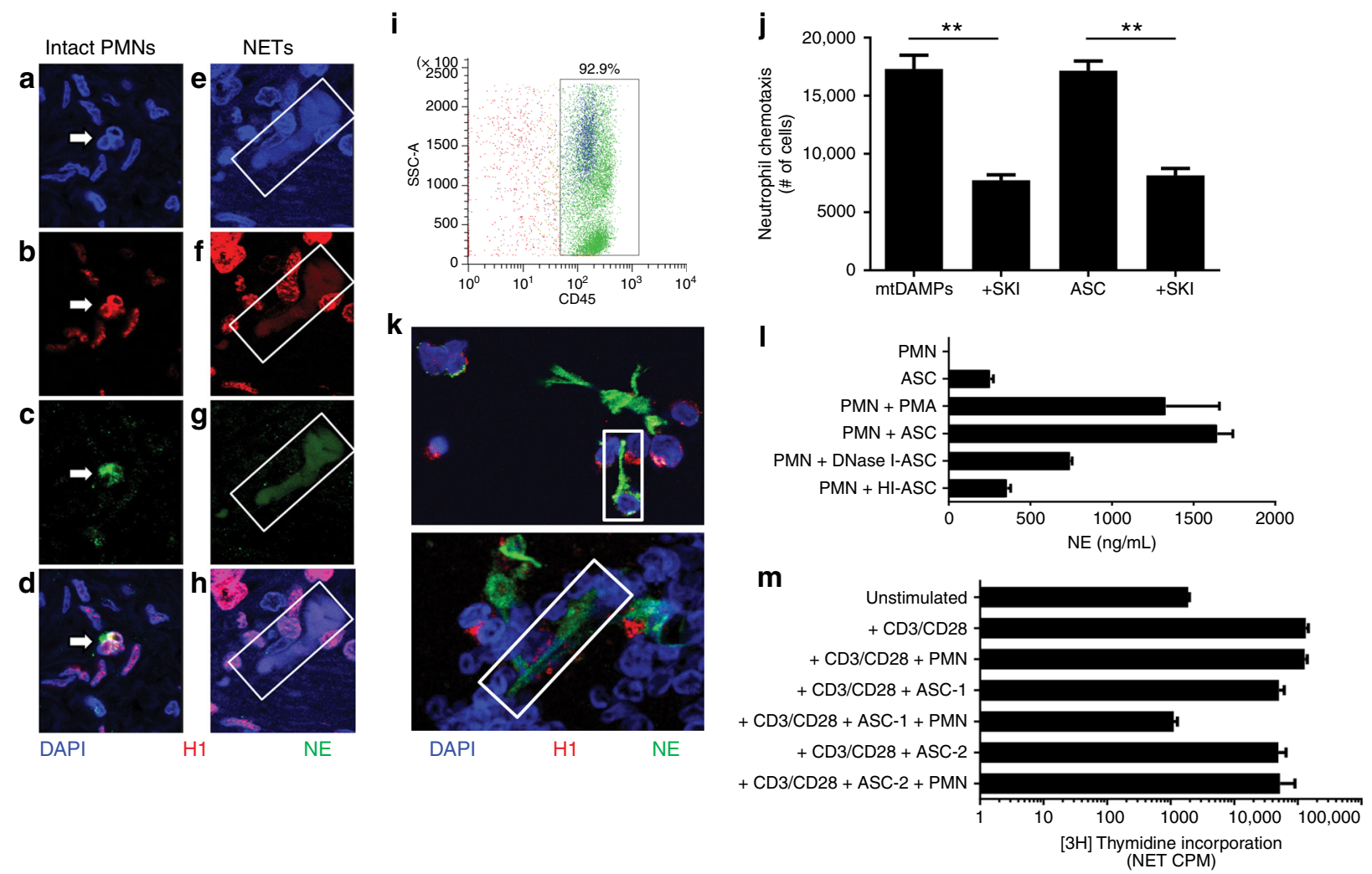

Fig. 2 Ovarian cancer ascites is chemoattractive to neutrophils and induces NETs and suppressive neutrophils. a-h Resected tumours were collected from patients with newly diagnosed advanced EOC $(n=5)$. Tumours were evaluated for the presence of (a-d) intact neutrophils (PMNs, white arrows), identified by a hypersegmented nucleus (DNA, blue; histone H1, red), and cytoplasmic NE (green), and (e-h) NETs, identified by co-localised extracellular DNA, histone $\mathrm{H} 1$, and NE (white boxes). PMNs within tumours were rare. NETs were visualised in 4/5 tumours evaluated. i Ascites were collected from patients with newly diagnosed HGSOC $(n=9)$. $>90 \%$ of the cells are CD45 leukocytes and on average $15-20 \%$ are granulocytic (blue). $\mathbf{j}-\mathbf{m}$ Ascites supernatants (ASC) were evaluated for pathways of PMN activation. PMNs were isolated from peripheral blood of healthy donors. $\mathbf{j}$ Donor PMN chemotaxis was measured in response to mtDAMPs (positive control) and ascites supernatants $(n=2)$. Sphingosine kinase inhibitor (SKI) was added to both mtDAMPs and ascites supernatants as a negative control to inhibit chemotaxis $\left({ }^{* *} p<0.01\right)$. k Donor PMNs were treated for $1 \mathrm{~h}$ with ascites supernatants and evaluated for NET generation (white boxes) by immunofluorescent confocal microscopy. Exposure of neutrophils to 3 of 4 ascites supernatants resulted in NETs. I Donor PMNs were treated for $1 \mathrm{~h}$ with media, $100 \mathrm{nM}$ PMA (positive control), ascites supernatants $(n=4)$, DNase I-pre-treated ascites supernatants, or heatinactivated ascites supernatants, and evaluated for degranulation by NE ELISA. Ascites-induced neutrophil degranulation, which was partially reversed with heat-inactivation (HI-ASC) or DNase I pre-treatment. $\mathbf{m}$ Autologous donor PMNs, $\mathrm{CD}^{+}$, and $\mathrm{CD}^{+}{ }^{+} \mathrm{T}_{\text {cells were isolated and used }}$ in co-culture. Neither PMNs nor ascites supernatants alone suppress anti-CD3/CD28-stimulated T cell proliferation, however, the majority of ascites supernatants from patients with newly diagnosed HGSOC $(n=17 / 22)$ induced PMNs to suppress T cell proliferation. ASC-1 is an example of an ascites sample that induced the neutrophil suppressor phenotype, and ASC-2 is an example of an ascites sample that did not induce the neutrophil suppressor phenotype. Data are from $\geq 3$ independent experiments

in vivo murine model, while the tumour microenvironment in EOC is characterised by persistent inflammation and injury. We observed fibrin aggregates, taken directly from the tumour microenvironment at surgery, embedded with neutrophils and tumour cells. Since fibrin adheres to surfaces, these fibrinneutrophil-tumour cell networks may be important for seeding of serosal surfaces, a characteristic feature of advanced EOC. Platelets can also increase the proliferation of EOC cells through TGF- $\beta$-dependent signalling. ${ }^{45}$ Taken together, these results suggest that therapeutic targeting of neutrophil-platelet interactions may inhibit EOC progression and metastasis.

One mechanism by which neutrophils might accelerate tumour progression is through cross signalling with platelets. Platelet-derived mtDNA and microparticles in EOC ascites may be a mechanism for cross-activation of neutrophils and NETosis. This idea is supported indirectly by our observation that ascites levels of mtDNA (but not gDNA since platelets are anucleate) correlated with worse prognosis. An important observation was the substantial variability observed in the ability of DNase I and protease inhibitors to prevent platelet activation by ascites supernatants, suggesting that multiple pathways exist for platelet activation in the tumour microenvironment. Similar to EOC ascites, ascites supernatants from patients with cirrhosis also activated platelets, supporting the notion that platelets are prone to activation outside of the circulation, particularly in response to an acute injury or inflammatory stimuli, and their responses are not specific to tumour-associated factors. EOC ascites is highly inflammatory and injurious and contains fibrin deposits harbouring neutrophils and tumour cells, while cirrhotic ascites is typically transudative with few inflammatory cells. Cirrhotic ascites contains several procoagulants, including but not limited to pre-activated Factor $X$ (Xa) and tissue factor. ${ }^{46}$ Tissue factor is also abundant in malignant pleural effusions from lung adenocarcinoma patients. ${ }^{47}$ Therefore, it is plausible that several procoagulants are present in all ascites, regardless of disease origin, and have the potential to activate platelets and thrombosis. Thus, in EOC, our data and results from others ${ }^{14,19}$ suggest that platelets are recruited to and activated in the 

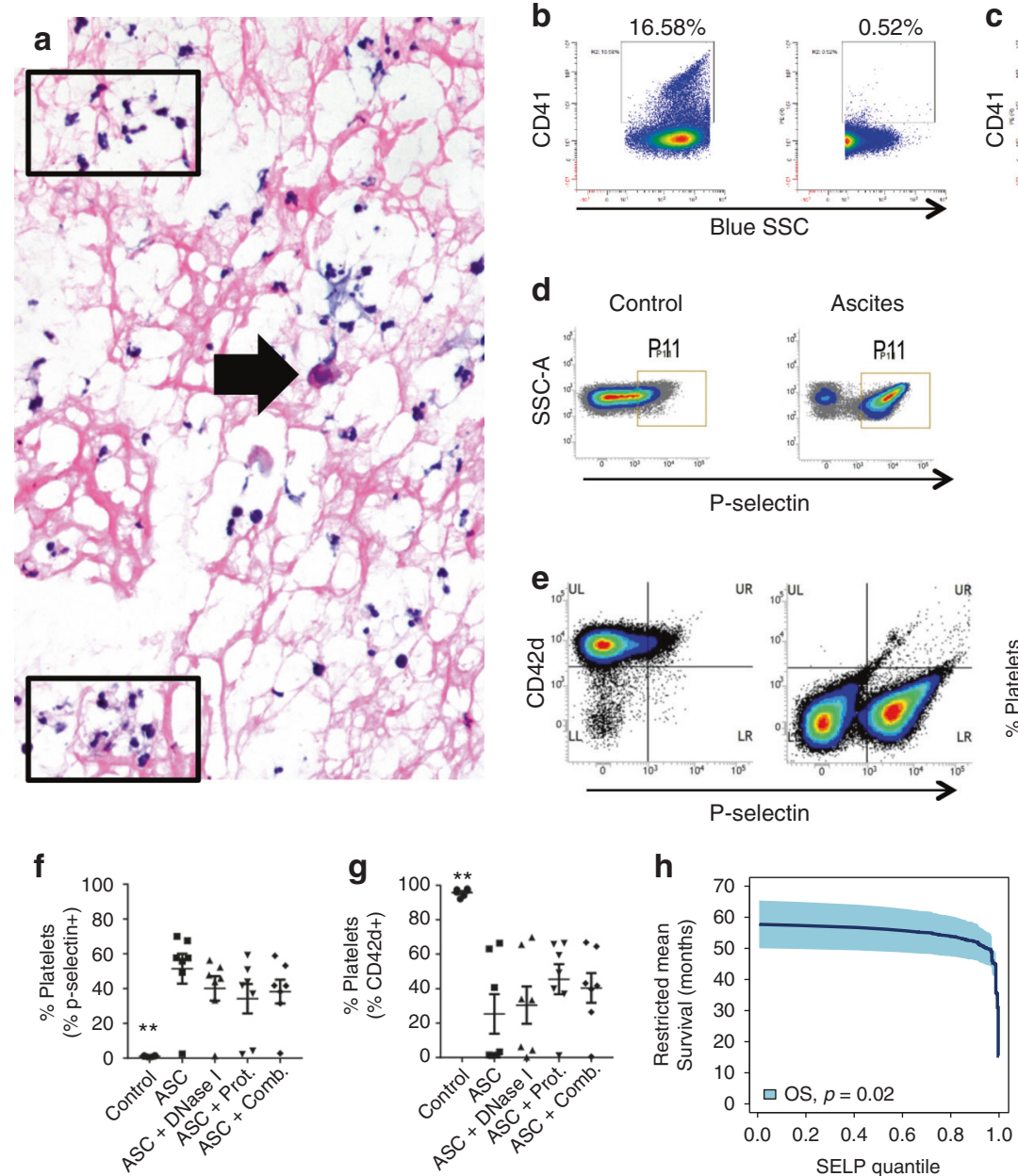
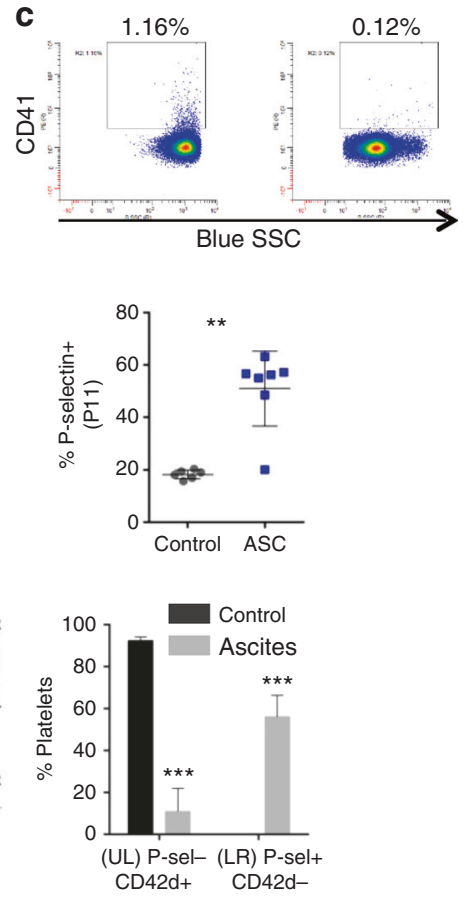

Fig. 3 Ovarian cancer ascites induces rapid platelet activation and aggregation that is partially abrogated by DNase and protease treatments. a-g Ascites were collected from patients with newly diagnosed advanced EOC and $500 \mathrm{~g}$ supernatants (ASC) were used. a Floating aggregates were collected from ascites before centrifugation and analysed by H\&E. Abundant neutrophils (black box) and a sparse number of tumour cells (black arrow) embedded in fibrin deposits (pink filaments) were identified. $\mathbf{b}, \mathbf{c}$ CD $41^{+}$PMP were measured in ascites supernatants by modified flow cytometry $(n=2)$. Variability exists between different patients (B, left) $16.58 \%$ and $\left(C\right.$, left) $1.16 \%$ CD41a ${ }^{+}$. In parallel, samples were $0.1 \mu \mathrm{m}$-filtered to remove microparticles as a specificity control (b, c, right panels). $\mathbf{d}-\mathbf{g}$. Platelets were isolated from peripheral blood of healthy donors and murine cardiac puncture. $\mathbf{d}$ Donor platelets were exposed to Tyrode's buffer with $1 \mathrm{mM} \mathrm{CaCl}$ (negative control) or ascites supernatants $(n=7)$ in the presence of $1 \mathrm{mM} \mathrm{CaCl}$ for 30 minutes prior to staining for flow cytometry. Representative density plots show increased number of P-selectin ${ }^{+}$platelets (P11 gate) after exposure to ascites supernatants, which is quantified to the right $(* *, p<0.01)$. E) Naïve murine platelets were exposed to the same ascites supernatants $(n=7)$, resulting in increased P-selectin expression and loss of CD42d from the surface of platelets within 15 minutes. The proportion of platelets in the upper left (UL; P-selectin ${ }^{-}$CD42 $\mathrm{d}^{+}$; resting) and lower right (LR; P-selectin ${ }^{+} \mathrm{CD} 42 \mathrm{~d}^{-}$; activated) quadrants of the density plots are quantified to the right $\left({ }^{* * *} p<0.001\right)$. f, g) After treatment with ascites supernatants with or without DNase I $(0.05 \% \mathrm{w} / \mathrm{v})$ and/or protease inhibitors (1:100), the proportions of F) P-selectin ${ }^{+}$donor platelets and (g) $\mathrm{CD}_{42} \mathrm{~d}^{+}$murine platelets are quantified. Data are from $\geq 3$ independent experiments. $\mathbf{h}$ High P-selectin mRNA expression in tumour is associated with reduced OS following primary surgery. TCGA provisional RNA Seq V2 RSEM data from cBioportal for ovarian serous cystadenocarcinoma $(n=302)$ were mined for expression of SELP. Quantiles of SELP expression were plotted against restricted mean survival (RMS) and SELP expression was associated with worse OS (Cox model, $p=0.02, \mathrm{HR}=1.14,95 \% \mathrm{Cl}: 1.03-1.28$ )

tumour microenvironment where they drive thrombosis and metastasis. In addition, extracellular histones, which can result from NETosis, are highly injurious ${ }^{48}$ and can promote thrombosis. $^{25}$ Histone $\mathrm{H} 1$ concentration was 10 -fold higher in ascites supernatants than $\mathrm{H} 3$, which may be due to NETs releasing extracellular $\mathrm{H} 1$. A limitation of these studies is that we cannot distinguish the source of extracellular histones, whether from neutrophils or other cells.

Considering the body of literature and our results on neutrophil-platelet interactions promoting tumour progression, we propose a model of EOC progression that links injury, neutrophilic inflammation, and thrombosis (Fig. 4). Cellular necrosis in the tumour microenvironment releases DAMPs (e.g., mtDNA), which recruit neutrophils. mtDNA within ascites stimulates NETosis, resulting in the release of proteases (e.g., $\mathrm{NE}$ and matrix metalloproteinases) that remodel extracellular matrix, and chromatin and tissue factor that promote platelet activation and thrombosis. While these responses serve to target microbes and prevent their dissemination, in the tumour microenvironment we posit that they enable tumour seeding of serosal surfaces where early metastasis in EOC occurs. In addition to the direct adhesive effect of fibrin, activated platelets cross signal with neutrophils and release pro-proliferative and angiogenic factors that enhance tumour progression and metastasis. In addition, ascites chemoattracts and induces a suppressor phenotype in neutrophils that is expected to be a barrier to tumour immunity. While strengths of our experimental approach include the use of primary cells and ascites as an 


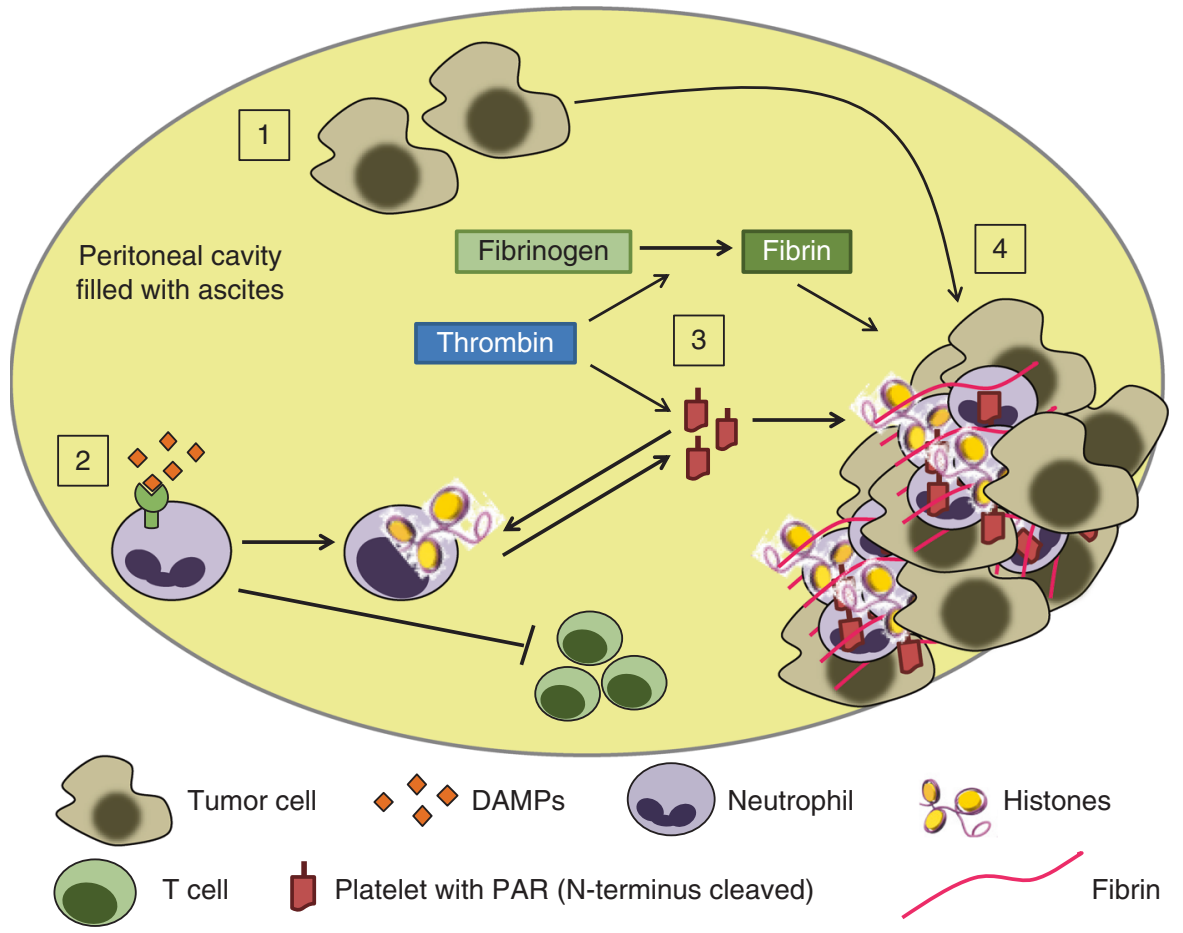

Fig. 4 Model of DAMPs and neutrophil-platelet responses in the ascites of patients with advanced EOC. (1) A hallmark of advanced cancer is cellular necrosis, which releases DAMPs, and minor numbers of tumour cells into the ascites. (2) mtDNA, and likely other DAMPs, recruit and activate neutrophils, induce NETosis, and (3) activate platelets. (4) Platelet activation and aggregation with NETs and fibrin filaments trapfree floating tumour cells and enhance seeding to the serosa and local dissemination within the peritoneal cavity. In addition, neutrophils acquire a T cell suppressor phenotype after ascites exposure, an effect that is predicted to impair T cell immunity and obstruct immunotherapy

authentic component of the EOC microenvironment, a limitation is that biological studies are ex vivo and don't encapsulate the complexity of the tumour microenvironment.

Our model includes a number of potentially targetable pathways involving neutrophil recruitment and activation, including NETosis, thrombosis, and impairment of $\mathrm{T}$ celldependent anti-tumour immunity. Inhibition of neutrophil recruitment (e.g., with small molecule CXCR2 inhibitors) and NETosis has shown benefit in murine tumour models. ${ }^{28,29,49}$ Coffelt et al. $^{50}$ showed in a mammary tumour model that tumour-induced neutrophils suppressed CTL responses, and depletion of IL-17 or G-CSF abrogated the T-cell suppressive phenotype of neutrophils. In addition, the absence of $\gamma \delta \mathrm{T}$ cells and neutrophils limited metastasis. McGray et al. ${ }^{51}$ recently showed that anti-Gr1 depletion enhanced the benefit of vaccination and PD-1 blockade in murine EOC. Here, we also observed that mtDNA activates DCs through TLR9-dependent and -independent pathways, an effect that may promote $T$ cell immunity. Thus, mtDNA and other DAMPs can have multiple effects on innate immune responses that can either accelerate or inhibit tumour progression.

We previously observed that granulocytes isolated from the ascites suppressed stimulated T cell proliferation ex vivo. ${ }^{31}$ In the current study, ascites supernatants induced a suppressor phenotype in neutrophils from healthy donors, characterised by the abrogation of proliferation of ex vivo stimulated autologous $T$ cells. It is well recognised that tumour-derived factors can induce a myeloid cell expansion in the marrow that leads to the generation of immature suppressive myeloid cells (i.e., MDSCs). However, this suppressive phenotype can also occur in activated neutrophils. ${ }^{52}$ Our results point to factors in the microenvironment of advanced EOC inducing a suppressor phenotype in mature neutrophils. Mechanisms for this suppression are complex and involve a number of neutrophil effector functions (Singel et al., manuscript under revision). Together with other suppressive pathways (e.g., MDSCs, tumour-associated macrophages, and regulatory $T$ cells), neutrophil-mediated suppression of $\mathrm{T}$ cell responses is expected to obstruct durable anti-tumour immunity.

Finally, although our results point to a pro-tumourigenic role of neutrophils in EOC, there is growing appreciation of neutrophil heterogeneity in cancer, with distinct neutrophil populations promoting cancer control or progression. It has been known for decades that activated neutrophils can kill tumour cells. More recently, Sagiv et al. ${ }^{53}$ identified distinct neutrophil populations in cancer with specific functional phenotypes and plasticity to switch between phenotypes. Knowledge about mechanisms by which the tumour microenvironment induces a suppressor phenotype in neutrophils may lead to new approaches to reprogram suppressive neutrophils to an anti-tumour phenotype. Our model points to products of injury, specifically mtDNA, and the subsequent neutrophil and platelet responses, as potential prognostic biomarkers and novel therapeutic targets in patients with advanced EOC. These results identified a number of neutrophilplatelet interactions and pathways to target to slow tumour progression.

\section{ACKNOWLEDGEMENTS}

Clinical data delivery, specimens, and honest broker services for this study were provided by the Clinical Data Network, Data Bank and BioRepository, and Pathology Resource Network, which are funded by the National Cancer Institute and are Roswell Park Comprehensive Cancer Center's Cancer Center Support Grant shared resources. We thank the Cell Biology Center (Flow Cytometry Core Facility) at the Institute of Experimental Medicine of the Hungarian Academy of Sciences, Budapest, Hungary for their assistance. Funding: This work was 
supported by Roswell Park Comprehensive Cancer Center grants $\mathrm{NCl}$ P30CA016056, the Roswell Park-UPCI Ovarian Cancer SPORE P50CA159981 (KO), NIH R01CA188900 (BHS AND KBM), T32CA108456 (KSG), T32CA085183 (KLS), and K01LM012100 (KHE). AD was supported by OTKA K109743, the Hungarian Brain Research Program KTIA_13_NAP-A-I/2, and the Momentum Program from the Hungarian Academy of Sciences and ERC-CoG 724994. The funders had no role in study design, data collection and analysis, decision to publish, or preparation of the manuscript.

\section{AUTHOR CONTRIBUTIONS}

All authors contributed extensively to the work presented in this paper. K.L.S., K.S.G., and B.H.S. developed the study and wrote the manuscript. K.L.S., K.S.G., A.N.H.K., M.J. G., A.C.D., P.C.M., T.R.E., N.L., R.F., Z.K., U.M., J.D.G., K.I., and A.D. designed and performed experiments, and analysed data. K.M. and K.H.E. analysed and described the statistical models, and contributed to the manuscript. B.H., C.F.U., and C.L. provided reagents and mice. J.D.G., C.J.H., K.B.M., K.O., A.D., and B.H.S. provided technical support and conceptual advice. All authors discussed the results and implications, and commented on the manuscript at all stages.Data availability:The data sets generated during and/or analysed during the current study are available from the corresponding author on reasonable request.

\section{ADDITIONAL INFORMATION}

Supplementary information is available for this paper at https://doi.org/10.1038/ s41416-018-0339-8.

Competing interests: The authors declare no competing interests.

Ethical approval: This study was approved by the Institutional Review Board of Roswell Park Comprehensive Cancer Center (Roswell Park), Buffalo, NY, and was in compliance with federal and state requirements. All participants gave informed consent to use samples for research (protocols i215512 and i188310). All studies were conducted in compliance with the Declaration of Helsinki. Mice were bred and maintained under specific pathogen-free conditions at the animal care facility at Roswell Park and used in compliance with all relevant laws and institutional guidelines under a protocol approved by the Institutional Animal Care and Use Committee.

\section{REFERENCES}

1. Winter, W. E. 3rd et al. Prognostic factors for stage III epithelial ovarian cancer: a Gynecologic Oncology Group Study. J. Clin. Oncol. 25, 3621-3627 (2007).

2. Jayson, G. C., Kohn, E. C., Kitchener, H. C. \& Ledermann, J. A. Ovarian cancer. Lancet 384, 1376-1388 (2014).

3. Zhang, L. et al. Intratumoral T cells, recurrence, and survival in epithelial ovarian cancer. N. Engl. J. Med. 348, 203-213 (2003).

4. Sato, E. et al. Intraepithelial CD8 + tumor-infiltrating lymphocytes and a high CD8 + /regulatory T cell ratio are associated with favorable prognosis in ovarian cancer. Proc. Natl Acad. Sci. USA 102, 18538-18543 (2005).

5. Curiel, T. J. et al. Specific recruitment of regulatory T cells in ovarian carcinoma fosters immune privilege and predicts reduced survival. Nat. Med. 10, 942-949 (2004).

6. Simpson-Abelson, M. R. et al. Human ovarian tumor ascites fluids rapidly and reversibly inhibit $T$ cell receptor-induced NF-kappaB and NFAT signaling in tumor-associated T cells. Cancer Immun. 13, 14 (2013).

7. Mhawech-Fauceglia, P. et al. Intraepithelial T cells and tumor-associated macrophages in ovarian cancer patients. Cancer Immun. 13, 1 (2013).

8. Obermajer, N., Muthuswamy, R., Odunsi, K. O., Edwards, R. \& Kalinski, P. PGE2dependent CXCL12 production and CXCR4 expression control the accumulation of human MDSCs in ovarian cancer environment. Cancer Res. 71, 7463-7470 (2011).

9. Lo, C. W. et al. IL-6 trans-signaling in formation and progression of malignant ascites in ovarian cancer. Cancer Res. 71, 424-434 (2011).

10. Kryczek, I. et al. B7-H4 expression identifies a novel suppressive macrophage population in human ovarian carcinoma. J. Exp. Med. 203, 871-881 (2006).

11. Kryczek, I. et al. Relationship between B7-H4, regulatory $\mathrm{T}$ cells, and patient outcome in human ovarian carcinoma. Cancer Res. 67, 8900-8905 (2007).

12. Cui, T. X. et al. Myeloid ${ }^{-}$derived suppressor cells enhance stemness of cancer cells by inducing microRNA101 and suppressing the corepressor CtBP2. Immunity 39, 611-621 (2013).
13. Sreeramkumar, V. et al. Neutrophils scan for activated platelets to initiate inflammation. Science 346, 1234-1238 (2014).

14. Labelle, M., Begum, S. \& Hynes, R. O. Platelets guide the formation of early metastatic niches. Proc. Natl Acad. Sci. USA 111, E3053-E3061 (2014).

15. Banerjee, S. et al. A multicenter, randomized trial of flat dosing versus intrapatient dose escalation of single-agent carboplatin as first-line chemotherapy for advanced ovarian cancer: an SGCTG (SCOTROC 4) and ANZGOG study on behalf of GCIG. Ann. Oncol. 24, 679-687 (2013).

16. Cho, H. et al. Pre-treatment neutrophil to lymphocyte ratio is elevated in epithelial ovarian cancer and predicts survival after treatment. Cancer Immunol., Immunother.: Cii. 58, 15-23 (2009).

17. Stone, R. L. et al. Paraneoplastic thrombocytosis in ovarian cancer. N. Engl. J. Med. 366, 610-618 (2012).

18. Lin, R. J., Afshar-Kharghan, V. \& Schafer, A. I. Paraneoplastic thrombocytosis: the secrets of tumor self-promotion. Blood 124, 184-187 (2014).

19. Haemmerle, M. et al. FAK regulates platelet extravasation and tumor growth after antiangiogenic therapy withdrawal. J. Clin. Invest. 126, 1885-1896 (2016).

20. Itagaki, K. et al. Mitochondrial DNA released by trauma induces neutrophil extracellular traps. PLOS ONE 10, e0120549 (2015).

21. Fuchs, T. A. et al. Novel cell death program leads to neutrophil extracellular traps. J. Cell Biol. 176, 231-241 (2007).

22. Caudrillier, A. et al. Platelets induce neutrophil extracellular traps in transfusionrelated acute lung injury. J. Clin. Invest. 122, 2661-2671 (2012).

23. Semeraro, F. et al. Extracellular histones promote thrombin generation through platelet-dependent mechanisms: involvement of platelet TLR2 and TLR4. Blood 118, 1952-1961 (2011).

24. Demers, M. et al. Cancers predispose neutrophils to release extracellular DNA traps that contribute to cancer-associated thrombosis. Proc. Natl Acad. Sci. USA 109, 13076-13081 (2012).

25. Martinod, K. et al. Neutrophil histone modification by peptidylarginine deiminase 4 is critical for deep vein thrombosis in mice. Proc. Natl Acad. Sci. USA 110, 8674-8679 (2013)

26. Stakos, D. A. et al. Expression of functional tissue factor by neutrophil extracellular traps in culprit artery of acute myocardial infarction. Eur. Heart J. 36, 1405-1414 (2015).

27. Etulain, J. et al. P-selectin promotes neutrophil extracellular trap formation in mice. Blood 126, 242-246 (2015).

28. Cools-Lartigue, J. et al. Neutrophil extracellular traps sequester circulating tumor cells and promote metastasis. J. Clin. Invest. 123, 67484 (2013).

29. Park, J. et al. Cancer cells induce metastasis-supporting neutrophil extracellular DNA traps. Sci. Transl. Med. 8, 361 ra138 (2016).

30. Kolomeyevskaya, N. et al. Cytokine profiling of ascites at primary surgery identifies an interaction of tumor necrosis factor-alpha and interleukin- 6 in predicting reduced progression-free survival in epithelial ovarian cancer. Gynecol. Oncol. 138, 352-357 (2015).

31. Khan, A. N. et al. Targeting myeloid cells in the tumor microenvironment enhances vaccine efficacy in murine epithelial ovarian cancer. Oncotarget 6 , 11310-11326 (2015).

32. Zhang, Q. et al. Circulating mitochondrial DAMPs cause inflammatory responses to injury. Nature 464, 104-107 (2010).

33. Rohm, M. et al. NADPH oxidase promotes neutrophil extracellular trap formation in pulmonary aspergillosis. Infect. Immun. 82, 1766-1777 (2014).

34. Eng, K. H., Schiller, E. \& Morrell, K. On representing the prognostic value of continuous gene expression biomarkers with the restricted mean survival curve. Oncotarget 6, 36308-36318 (2015).

35. Eng, K. H. \& Seagle, B. L. Covariate-adjusted restricted mean survival times and curves. J. Clin. Oncol. 35, 465-466 (2017).

36. Boone, B. A. et al. The receptor for advanced glycation end products (RAGE) enhances autophagy and neutrophil extracellular traps in pancreatic cancer. Cancer Gene. Ther. 22, 326-334 (2015).

37. Berger-Achituv, S. et al. A proposed role for neutrophil extracellular traps in cancer immunoediting. Front. Immunol. 4, 48 (2013).

38. Arai, Y. et al. Uric acid induces NADPH oxidase-independent neutrophil extracellular trap formation. Biochem. Biophys. Res. Commun. 443, 556-561 (2014).

39. Hakkim, A. et al. Activation of the Raf-MEK-ERK pathway is required for neutrophil extracellular trap formation. Nat. Chem. Biol. 7, 75-77 (2011).

40. Boilard, E. et al. Platelets amplify inflammation in arthritis via collagen-dependent microparticle production. Science 327, 580-583 (2010).

41. Duchez, A. C. et al. Platelet microparticles are internalized in neutrophils via the concerted activity of 12-lipoxygenase and secreted phospholipase A2-IIA. Proc. Natl Acad. Sci. USA 112, E3564-E3573 (2015).

42. Szender, J. B. et al. Impact of ascites volume on clinical outcomes in ovarian cancer: A cohort study. Gynecol. Oncol. 146, 491-497 (2017). 
Mitochondrial DNA in the tumour microenvironment activates neutrophils... $\mathrm{KL}$ Singel et al.

43. Naldini, A. et al. Identification of thrombin-like activity in ovarian cancer associated ascites and modulation of multiple cytokine networks. Thromb. Haemost. 106, 705-711 (2011).

44. Zhang, T. et al. Thrombin facilitates invasion of ovarian cancer along peritoneum by inducing monocyte differentiation toward tumor-associated macrophage-like cells. Cancer Immunol., Immunother.: Cii. 59, 1097-1108 (2010).

45. Cho, M. S. et al. Platelets increase the proliferation of ovarian cancer cells. Blood 120, 4869-4872 (2012)

46. Phillips, L. L. \& Rodgers, J. B. Procoagulant activity of ascitic fluid in hepatic cirrhosis: in vivo and in vitro. Surgery 86, 714-721 (1979).

47. Yeh, H. H. et al. Upregulation of tissue factor by activated Stat 3 contributes to malignant pleural effusion generation via enhancing tumor metastasis and vascular permeability in lung adenocarcinoma. PLOS ONE 8, e75287 (2013).

48. $\mathrm{Xu}$, J. et al. Extracellular histones are major mediators of death in sepsis. Nat. Med. 15, 1318-1321 (2009)

49. Steele, C. W. et al. CXCR2 inhibition profoundly suppresses metastases and augments immunotherapy in pancreatic ductal adenocarcinoma. Cancer Cell. 29, 832-845 (2016).

50. Coffelt, S. B. et al. IL-17-producing gammadelta $T$ cells and neutrophils conspire to promote breast cancer metastasis. Nature 522, 345-348 (2015).

51. Mcgray, A. R. et al. (eds.). Abstract 4557: Tumor immune profiling identifies multiple unique therapeutic targets that improve vaccination +oncolytic virotherapy against metastatic ovarian cancer. In: AACR Annual Meeting; 2017. American Association for Cancer Research, Washington, D.C. (2017).

52. Schmielau, J. \& Finn, O. J. Activated granulocytes and granulocyte-derived hydrogen peroxide are the underlying mechanism of suppression of $\mathrm{t}$-cell function in advanced cancer patients. Cancer Res. 61, 4756-4760 (2001).

53. Sagiv, J. Y. et al. Phenotypic diversity and plasticity in circulating neutrophil subpopulations in cancer. Cell Rep. 10, 562-573 (2015).

Open Access This article is licensed under a Creative Commons Attribution 4.0 International License, which permits use, sharing, adaptation, distribution and reproduction in any medium or format, as long as you give appropriate credit to the original author(s) and the source, provide a link to the Creative Commons license, and indicate if changes were made. The images or other third party material in this article are included in the article's Creative Commons license, unless indicated otherwise in a credit line to the material. If material is not included in the article's Creative Commons license and your intended use is not permitted by statutory regulation or exceeds the permitted use, you will need to obtain permission directly from the copyright holder. To view a copy of this license, visit http://creativecommons. org/licenses/by/4.0/.

(c) The Author(s) 2018 